\title{
No good evidence to link toothbrushing trauma to gingival recession
}

\author{
Is toothbrushing effective in preventing development or progression of \\ noninflammatory, localised gingival recession?
}

\author{
Rajapakse PS, McCracken GI, Gwynnett E, Steen ND, Guentsch \\ A, Heasman PA. \\ Does tooth brushing influence the development and progression of \\ non-inflammatory gingival recession? A systematic review. J Clin \\ Periodontol 2007; 34:1046-1061
}

Data sources Medline, Embase, Web of Knowledge, the Cochrane Central Register of Controlled Trials, Current Contents Connect and the Google Scholar databases were searched. Searches by hand were also made of the Journal of Clinical Periodontology, Journal of Periodontal Research and the Journal of Periodontology. Bibliographies of narrative reviews, conference proceedings and relevant texts known to the authors were also searched.

Study selection Screening of studies was carried out independently by three reviewers and assessed for quality. Studies of Level III, ie, observational studies without control groups (cross sectional studies, beforeand-after studies, case series) or better were included. Studies reported in the English, German, Spanish or Greek language were included.

Data extraction and synthesis Data were extracted from the full-text articles using a purposely designed data extraction form, and a qualitative summary was carried out.

Results Eighteen studies were included. One abstract from the meeting EuroPerio 5 reported a randomised-controlled clinical trial (RCT; Level I evidence) in which the authors concluded that the toothbrushes significantly reduced recessions on buccal tooth surfaces over the course of 18 months. Of the remaining 17 observational studies, two concluded there was no apparent relationship between toothbrushing frequency and gingival recession. Eight studies reported a positive association between toothbrushing frequency and recession. Other potential risk factors were duration of toothbrushing, brushing force, and frequency of changing the toothbrush, brush (bristle) hardness and toothbrushing technique. None of the observational studies satisfied all the specified criteria for quality appraisal, and a valid appraisal of the quality of the RCT was not possible.

Conclusions The data to support or refute the association between toothbrushing and gingival recession are inconclusive.

Address for correspondence: Professor Peter Heasman, School of Dental Sciences. University of Newcastle Framlington Place, Newcastle upon Tyne NE2 4BW, UK. E-mail: p.a.heasman@newcastle.ac.uk

\section{Commentary}

If you look in any periodontal textbook for the aetiology of gingival recession, you will probably find that gingival trauma and gingival abrasion from toothbrushing is believed to progress directly to gingival recession. Yet where is the evidence to support this long-held claim? Case studies documenting recession from toothbrush trauma are speculative, at best. Observational studies, particularly those that are cross-sectional in nature, suggest that gingival trauma and gingival abrasion may result from toothbrushing, but a direct relationship between traumatic home dental care and gingival recession cannot be made.

Rajapske and colleagues have conducted a very thorough systematic review of the literature. Their findings illuminate several issues of interest to researchers and the clinicians who rely on the results of clinical research. The most obvious conclusion is that, once again, we find the quality of research sadly lacking. Researchers must begin to pay heed to CONSORT guidelines (www.consort-statement.org/ ?o=1011) when designing clinical trials if we want good quality evidence with minimal bias and error.

The highest level of evidence Rajapske et al. found to answer their clinical question was a single abstract of an industry-sponsored RCT. The study found no difference in the amount of recession caused by manual or powered toothbrushes. Interestingly enough, 2 years after presentation at a scientific conference, the full trial has not been published. Whatever the possible reasons for this, as pointed out by the authors of the review, relying on the results of an abstract alone does not allow us to judge the amount or types of bias that may have been present in the study itself.

Finally, the findings of the systematic review speak to the fact that not everything can be subject to a RCT. In order to definitively conclude that toothbrushing itself is a cause of noninflammatory gingival recession, we would have to design a trial of adequate sample size, consisting of subjects of varying ages, with various tissue biotypes, and including smokers and nonsmokers - none of whom had recession to being with - and follow them over a long enough period of time to determine if recession does occur. Given the minimal morbidity associated with recession, it is unlikely that such a study would be funded.

The review provides some guidance as to how to design future research to best answer this question. As clinicians, we are again left to rely on common sense and clinical judgment, and hope good research is on the horizon.

\section{Debora C Matthews}

Division of Periodontics, Faculty of Dentistry, Dalhousie

University, Dalhousie, Halifax, Nova Scotia, Canada

Evidence-Based Dentistry (2008) 9, 49. doi:10.1038/sj.ebd.6400582 